\title{
Chronische muskuloskelettale Schmerzen in der Schweiz: Defizite in der Betreuungsqualität und Massnahmen für die Praxis
}

\section{B. A. Michel ${ }^{a}$, U. Tschumi ${ }^{b}$ \\ Ch. Beglinger, G. Dalvit ${ }^{d}$, \\ M. Felder', A. Graft, \\ J. Steurer ${ }^{g}$}

a Rheumaklinik und Institut für Physikalische Medizin UniversitätsSpital Zürich

b Dr. Schlegel Healthworld AG

c Abteilung Gastroenterologie und Hepatologie Universitätsspital Basel

d Ehemalige Leiterin der Schweizerischen Rheumaliga

e Spezialarzt FMH für physikalische Medizin und Rehabilitation, speziell Rheumatologie

f Praxis für Allgemeinmedizin Falkenstrasse Zürich

g Horten-Zentrum für praxisorientierte Forschung und Wissenszentrum UniversitätsSpital Zürich
Die Arbeiten der Arthritis Action Group (AAG) werden sowohl national wie international unterstützt durch einen Educational Grant der Firma Pfizer AG. Die AAG behält dabei die uneingeschränkte Kontrolle über die Studiendaten, die Interpretation und die Weitergabe der Studienergebnisse.

Korrespondenz:

Prof. Dr. med. Beat Michel Rheumaklinik

UniversitätsSpital Zürich

Gloriastrasse 25

CH-8091 Zürich

Tel. 0442552970

Beat.michel@usz.ch

\section{Defizite in der Betreuungsqualität}

Rund 15\% der Schweizer Bevölkerung leiden unter chronischen muskuloskelettalen Schmerzen (CMS). Dieses Resultat lieferte eine internationale Studie mit Einbezug der Schweizer Bevölkerung sowie von Schweizer Ärzten [1]. Diese Erhebung zeigte in allen acht untersuchten europäischen Ländern erhebliche Defizite in der Betreuung von Patienten mit CMS.

Für die Schweiz wurden folgende Mängel festgestellt: Bei 35\% der Fälle wurde keine Diagnose gestellt bzw. der Patient kannte seine Diagnose nicht; die Lebensqualität ist bei einem Drittel der Patienten deutlich reduziert. Patienten sind über ihre Erkrankung schlecht orientiert und die medikamentöse Behandlung wird oft inadäquat beurteilt.

Eine ergänzende Untersuchung mittels Workshops sowohl bei Ärzten als auch bei Patienten bestätigte diese Schwachpunkte und deckte speziell folgende Mängel auf: Die genauen Ursachen der Schmerzen bleiben oft unklar, es bestehen teils erhebliche Kommunikationsprobleme zwischen Patient und Arzt, der Umgang mit der medikamentösen Therapie erfolgt oft inadäquat, und schliesslich besteht ein mangelhaftes Zeitmanagement während der Konsultation. Die Arthritis Gruppe Schweiz (zusammengesetzt aus den Autoren dieses Artikels) setzte sich aufgrund der gefundenen Defizite die Aufgabe, Massnahmen zur Verbesserung der Betreuung von Patienten mit CMS in der Praxis zu entwickeln.

\section{Möglichkeiten der Verbesserung - unterstützende Massnahmen für die Praxis} Basierend auf der Erhebung der europäischen Arthritis Action Group hat die Arthritis Action Group Schweiz ab 2003 Interviews und Workshops mit Betroffenen und Ärzten durchgeführt, um geeignete Massnahmen zur Verbesserung der Situation von Betroffenen abzuleiten. Die Auswertung ergab insbesondere Defizite in der Diagnostik, in der Kommunikation zwischen Patient und Arzt und in der Anwendung medikamentöser Therapien. Als Verbesserungsmassnahmen erarbeitete die AAG Schweiz in der Folge interaktive Fortbildungsmodule zu den Schwerpunkten «spezifischer/unspezifischer Rückenschmerz», «lumbale Diskushernie» und «enger Spinalkanal». Zur Unterstützung der Diagnostik von Rückenschmerzen wurde von «Rheuma Schweiz» in Zusammenarbeit mit der AAG

\section{Douleurs musculo-squelettiques}

chroniques en Suisse: déficits dans

la qualité des soins et mesures

à prendre pour la pratique

Une étude européenne comprenant aussi la Suisse a prélevé des déficits considérables en ce qui concerne la prise en charge des patients avec des douleurs musculo-squelettiques chroniques: $35 \%$ des cas n'avait pas été diagnostiqué, pour $1 / 3$ des patients la qualité de vie était nettement réduite, les patients étaient mal informés sur leur maladie et le traitement médicamenteux était souvent jugé de manière inadéquate. Plusieurs investigations en Suisse ont confirmé ces résultats et ont montré des points faibles importants: La cause des douleurs souvent n'est pas claire, il y a des problèmes de communication entre patient et médecin, la gestion du temps pendant la consultation est sous-optimale.

Sur la base de ces résultats I'Arthritis Action Group Suisse a réalisé plusieurs projets afin d'instaurer des mesures destinées à améliorer la situation. Elle a réalisé, dès 2003, des interviews et des workshops avec des médecins et des personnes atteintes. L'évaluation a montré, en particulier, des déficits au niveau du diagnostic, de la communication entre patient et médecin ainsi que de l'emploi de thérapies médicamenteuses. Suite à ce constat, l'AAG Suisse a élaboré des mesures d'amélioration, à savoir des modules interactifs de formation continue sur les points forts «douleurs dorsales spécifiques/non spécifiques», «hernie discale lombaire» et «canal spinal étroit». Pour soutenir le diagnostic, «Rheuma Suisse» a développé un logiciel en collaboration avec I'AAG Suisse. De plus, sur l'initiative du groupe, des patients spécialement formés - les «Patient Partners» - se tiennent à disposition des médecins intéressés pour l'entraînement intense de la communication médecin-patient. 
Schweiz eine Software entwickelt, und zum intensiven Trainieren der Arzt-Patienten-Kommunikation stehen interessierten Ärzten heute auf Initiative der AAG Schweiz speziell ausgebildete Patienten zur Verfügung: Patient Partners.

Die Gruppe der chronischen muskuloskelettalen Schmerzzustände (CMS) bereitet in der Praxis immer wieder Probleme: Die heterogenen Krankheitsbilder mit ausgeprägter interindividueller Variabilität sind vielfach nicht einfach zu diagnostizieren, und die Patienten werden oft inadäquat betreut [1]. Um diese Situation besser zu untersuchen und Massnahmen zur Verbesserung der Patientenbetreuung abzuleiten, wurde die internationale europäische Arthritis Action Group (AAG) ins Leben gerufen. Eine fachlich ähnlich zusammengesetzte Gruppe in der Schweiz nahm die Aufgabe wahr, die Situation in der Schweiz spezifisch zu evaluieren und die angemessen erscheinenden Massnahmen daraus abzuleiten und umzusetzen. Die Arthritis Action Group Schweiz setzt sich zusammen aus je einem Allgemeinmediziner (A. Graf), Internisten (J. Steurer) und Gastroenterologen (Ch. Beglinger), aus zwei Rheumatologen (M. Felder, B. Michel) und einer Vertreterin der Rheumaliga Schweiz (G. Dalvit) [2].

\section{Die identifizierten Defizite in der Versorgung}

Die Auswertung der Schweizer Daten bzw. der geführten Interviews und Workshops mit Betroffenen zeigte hauptsächlich Handlungsbedarf in vier Bereichen. Die Angaben der Ärzte und Patienten waren nahezu deckungsgleich:

- Die Diagnose von muskuloskelettalen Schmerzen ist sehr schwierig. Die Krankheitsbilder sind unklar und sehr patientenabhängig.

- Es bestehen weitreichende Kommunikationsprobleme zwischen Patient und Arzt.

- Die medikamentöse Therapie wird oft inadäquat angewendet.

- Die Konsultationen sind geprägt vom Zeitmangel des Arztes im Praxisalltag, bzw. von mangelhaftem Zeitmanagement.

Die AAG Schweiz definierte nach diesen Erkenntnissen mehrere Massnahmenpakete, die einerseits das Fachwissen der Ärzte verbessern, andererseits das anspruchsvolle Thema der Arzt-Patienten-Beziehung aufgreifen und optimieren wollen. Erarbeitet wurden diese Massnahmen in interdisziplinären Teams, um möglichst alle Aspekte einbeziehen zu können.

\section{Die Massnahmen}

\section{Fortbildungs-Module: Fachwissen}

\section{kompakt und effizient vermittelt}

Sehr häufig in der Arztpraxis sind Rückenschmerzen. Mehrheitlich kann dafür eine Ursache gefunden werden, z. B. Diskushernien oder ein zu enger Spinalkanal.
Diese beiden häufigen Ursachen von Rückenschmerzen werden deshalb auch in je einem FortbildungsModul der Arthritis Action Group aufgegriffen. Ein drittes Modul widmet sich den Rückenschmerzen unklarer Ursache. Zielgruppe sind Hausärzte und Internisten in der Praxis. Interessierten Rheumatologen bietet sich damit die Möglichkeit, als Referent die Zusammenarbeit mit den Hausärzten ihrer Region zu festigen und sich auf gemeinsame Vorgehensweisen in Diagnose und Behandlung zu einigen. Die drei Module eignen sich sehr gut für Veranstaltungen in kleinem Rahmen, z. B. also für Qualitätszirkel von Ärztenetzwerken.

\section{Modul 1: Spezifischer versus} unspezifischer Rückenschmerz

Vor dem Hintergrund, dass 90\% der Schmerzen im Rücken erklärbar sind, vermittelt die Lerneinheit streng praxisorientiert anhand eines konkreten Patientenfalls das Wissen über die erforderlichen diagnostischen Schritte, um verschiedene, häufig auftretende Erscheinungsformen des Rückenschmerzes einzuschätzen und abzuklären. Relevante Untersuchungsmethoden werden anschaulich demonstriert. Das Lernmodul formuliert Behandlungsziele und bezieht dazu auch stark die Patientensicht ein. Die therapeutischen Überlegungen gliedern sich in kurzzeitige und langzeitige Massnahmen und geben Hinweise zur Prognose. Konzipiert ist das Slidekit für eine Arbeitsgruppe von ca. 8-12 Hausärzten. Sie wird geleitet durch einen Rheumatologen, wobei nach Bedarf ein Physiotherapeut beigezogen werden kann.

\section{Modul 2: Rückenschmerzen bei lumbaler} Diskushernie und bei engem Spinalkanal

Auch diese Fortbildungseinheit geht anhand eines Praxisfalles auf die notwendigen Schritte zur Diagnostik und Therapie ein. Ausgehend von einer Übersicht der verschiedenen Wirbelsäulensyndrome

- (lumbo-)vertebrale Syndrome,

- spondylogene Syndrome (nerval, vasal, tendomyogen),

- Kompressionssyndrome (radikulär, medullär, vaskulär),

führen die Fachreferenten die Teilnehmer anhand zweier Patientenfälle anschaulich durch alle relevanten Stationen der Diagnostik, Therapie und Nachbetreuung. Dieses Modul ist ebenfalls für die intensive Arbeit in Kleingruppen von 8-12 Hausärzten konzipiert.

Beide Fortbildungseinheiten wurden als Pilotworkshops durchgeführt und evaluiert. Die Teilnehmer bescheinigten den beiden Veranstaltungen eine sehr hohe fachliche Kompetenz und grosse Praxisnähe. Umfang und Stoffdichte wurden als angemessen beurteilt. Das Konzept der Kleingruppe wurde als unterstützend und anregend bezeichnet. 
Computer Aided Diagnosis: Abklärung von Rückenschmerzen

Kreuzschmerzen sind eines der häufigsten Symptome, mit denen sich der Hausarzt konfrontiert sieht. Dahinter können viele verschiedene Ursachen versteckt sein. Die diagnostische Zuordnung beruht vorwiegend auf den Angaben des Patienten und der klinischen Untersuchung des Arztes. Unter Federführung von «Rheuma Schweiz» [3] wurde in Zusammenarbeit mit der AAG Schweiz ein Computer-Programm entwickelt, das die Abklärungsschritte im Sinne einer praxisrelevanten Hilfestellung systematisch abbildet [4]. Dadurch ergeben sich für die Software Einsatzgebiete wie: 1. Selbständige Fortbildungsmöglichkeit zum Thema Kreuzschmerzen, 2. Methodische und didaktische Unterstützung eines Fachreferenten bei Vorträgen zur Thematik Kreuzschmerzen, 3. Anwendung als Experten-System für komplexe Fälle von Kreuzschmerzen.

In der Software können Patientenbeschwerden und klinische Untersuchungsbefunde erfasst werden, woraus automatisch eine Liste von Differentialdiagnosen generiert wird. Der Auswertungsalgorithmus basiert auf einer Datenbank häufiger Ursachen von Kreuzschmerzen, angereichert mit von Experten verfassten Regeln zur Diagnose, Differentialdiagnose und Therapie. Auch das Erkennen von Notfallsituationen bei Patienten mit akuten Kreuzschmerzen ist durch diese Diagnose-Software möglich.

Entscheidende Untersuchungstechniken der Wirbelsäule sowie wichtige neurologische Tests sind als Video-Sequenzen integriert, was eine selbständige Entwicklung und Verfeinerung der eigenen Untersuchungstechnik ermöglicht. Die erste Qualitätssicherung des Systems erfolgte durch die erfolgreiche Eingabe von realen Patientendaten sowie im Einsatz als Fortbildungsinstrument in Workshops mit Praktikern.

\section{Patient Partners: So denken Ihre Patienten}

Die Kommunikation mit CMS-Patienten ist nicht einfach: Jeder fühlt anders, drückt sich anders aus, und gerade wenn objektive Methoden wie Röntgen, CT oder MRI Fragen offen lassen, ist die Anamnese ein besonders wichtiges diagnostisches Instrument. Die Erhebungen der Arthritis Action Group Schweiz zeigten, dass gerade in diesem Bereich Optimierungspotential liegt. Seit 2006 führt die Arthritis Action Group Schweiz deswegen «Patient Partners-Workshops» durch [5]. In diesen akkreditierten Fortbildungen vermitteln geschulte Arthritis-Patientinnen und -patienten einer kleinen Gruppe von Hausärzten ihre Erfahrungen und ihr Wissen. Durch direkte Interaktion zwischen Arzt und Patient werden alle Teilnehmer aktiv integriert. Mit dieser neuartigen Form der ärztlichen Fortbildung wird die Sensibilisierung der Hausärzte für eine rheumatische Erkrankung und eine optimale Kommunikation mit Patienten gefördert, indem das Wissen über bzw. das Leben mit der Krankheit von direkt Betroffenen vermittelt wird - diese Erfahrung ist in keinem Lehrbuch zu finden.

Die Vorbereitung für die Einsätze als «Patient Partner» beinhaltet neben einem rheumatologischen Grundkurs auch den Austausch mit anderen Betroffenen. So demonstrieren «Patient Partners» beispielsweise, wie man eine rheumatische Hand untersucht, und geben die Sichtweise der Patienten bezüglich Diagnosestellung, Behandlung und Erfahrungen im Alltag weiter. Diese Erkenntnisse sollen den Hausarzt sensibilisieren und dabei helfen, die Zeitspanne zwischen Erstkontakt und Diagnosestellung zum Wohl des Patienten zu verkürzen. Die Evaluation erster Workshops hat gezeigt, dass die Hausärzte diese neue Art von Fortbildung annehmen, schätzen und gerne weiterempfehlen. In der Deutschschweiz gibt es zurzeit 10 «Patient Partners», in der Romandie 6 und im Tessin einen. Sie haben bereits 14 Workshops zur Indikation Rheumatoide Arthritis durchgeführt.

\section{Ausblick der AAG Schweiz}

Mit den beschriebenen Massnahmen zur Verbesserung der Betreuung von Patienten mit CMS konnte die AAG Schweiz erste gute Erfahrungen und positive Rückmeldungen von den Teilnehmern sammeln. Ziel der AAG Schweiz ist es nun, diese Massnahmen breit zugänglich und nutzbar zu machen. Interessenten erhalten weitere Informationen dazu bei der Dr. Schlegel Healthworld AG (info@ischlegelhealth.ch). Aufgrund der guten bisherigen Erfahrungen wird die AAG Schweiz weitere Anstrengungen zur Erarbeitung zusätzlicher Massnahmen unternehmen, welche die erkannten Defizite in der Betreuung von Patienten mit CMS reduzieren helfen.

\section{Literatur}

1 Woolf AD, Zeidler H, Haglund U et al. Musculoskeletal pain in Europe: Its impact and a comparison of population and medical perceptions of treatment in eight European countries. Ann Rheum Dis. 2004;63:342-7.

2 Michel BA, Tschumi U, Woolf AD et al. Chronische muskuloskelettale Schmerzen in der Schweiz: Die Betreuungsqualität aus Sicht der Ärzte und Patienten. Praxis Schweizerische Rundschau für Medizin. 2009;17.

3 Rheuma-Schweiz®: www.rheuma-schweiz.ch

4 Medlink: www.med-link.ch

5 Patient Partners: «The Patient Partners programme is the only global, patient-led educational programme of its kind and is designed to help improve physicians' ability to diagnose and manage patients with arthritis.» (www.patient-partners.org). Vertretung Patient Partners Schweiz: cg@cg-empowerment.ch 